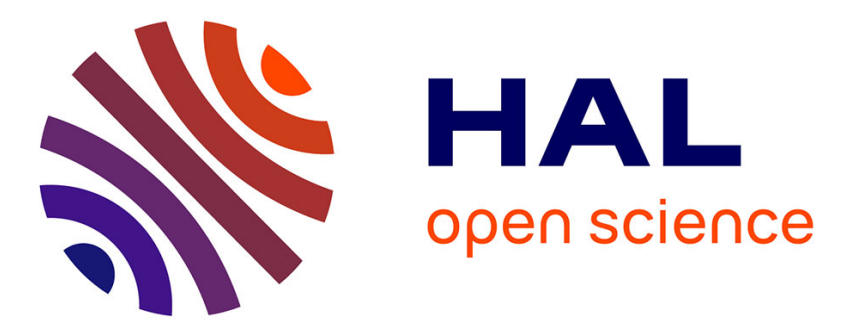

\title{
Behaviors classification based distance measuring system for pedestrians via a foot-mounted inertial sensor
}

\author{
Zebo Zhou, Shanhui Mo, Jin Wu, Hassen Fourati
}

\section{To cite this version:}

Zebo Zhou, Shanhui Mo, Jin Wu, Hassen Fourati. Behaviors classification based distance measuring system for pedestrians via a foot-mounted inertial sensor. Asian Journal of Control, 2019, 21 (4), pp.1483-1495. 10.1002/asjc.2005 . hal-01929624

\section{HAL Id: hal-01929624 \\ https://hal.inria.fr/hal-01929624}

Submitted on 21 Nov 2018

HAL is a multi-disciplinary open access archive for the deposit and dissemination of scientific research documents, whether they are published or not. The documents may come from teaching and research institutions in France or abroad, or from public or private research centers.
L'archive ouverte pluridisciplinaire HAL, est destinée au dépôt et à la diffusion de documents scientifiques de niveau recherche, publiés ou non, émanant des établissements d'enseignement et de recherche français ou étrangers, des laboratoires publics ou privés. 


\title{
Behaviors classification based distance measuring system for pedestrians via a foot-mounted inertial sensor
}

\author{
Zebo Zhou, Shanhui Mo, Jin Wu and Hassen Fourati
}

\begin{abstract}
In this paper, we develop a foot-mounted pedestrian navigation system prototype with the emphasis on distance measuring with an inertial measurement unit (IMU) which implies the characteristics of pedestrian gait cycle and thus can be used as a crucial step indicator for distance calculation. Conventional methods for step detection and step length estimation cannot adapt well to the general pedestrian applications since the parameters in these methods may vary for different persons and motions. In this paper, an adaptive time- and frequency-domains joint distance measuring method is proposed by utilizing the means of behaviors classification. Two key issues are studied: step detection and step length determination. For the step detection part, first behavior classification along with state transition strategy is designed to identify typical pedestrian behaviors including standing still, walking, running and irregular swing. Then a four-stage step detection method is proposed to adaptively determine both step frequency and threshold in a flexible window. Based on the behavior classification results, a two-segment functional based step length model is established to adapt the walking and running behaviors. Finally, real experiments are carried out to verify our proposed step detection method and step length model. The results show that our method outperforms the existing representative methods and it exhibits the merits of accuracy and adaptability for different persons in real time and significantly improves the accuracy of distance measuring.
\end{abstract}

Key Words: pedestrian navigation; step detection; step length estimation; behavior classification; state machine

\section{INTRODUCTION}

Correspondent: Z. Zhou is with School of Aeronautics and Astronautics, University of Electronic Science and Technology of China, Chengdu, China. E-mail: klinsmann.zhou@gmail.com.

S. Mo is with School of Aeronautics and Astronautics, University of Electronic Science and Technology of China, Chengdu, China. E-mail: rubyhui.mo@gmail.com.

J. Wu is with Department of Electronic \& Computer Engineering, Hong Kong University of Science and Technology, Hong Kong, China. E-mail: jin_wu_uestc@ hotmail.com

H. Fourati is with the University Grenoble Alpes, CNRS, GIPSA-Lab, Grenoble 38400, France, and also with Inria, Grenoble. E-mail: hassen.fourati@gipsa-lab.grenoble-inp.fr.
With the increasing demands of location based services (LBS), the pedestrian navigation system (PNS) has gained widespread attentions [1]. The global navigation satellite system (GNSS) can achieve satisfactory positioning accuracy for outdoor pedestrians. However, it is not necessarily the case for challenging environments, e.g. indoors, city canyons, signal jamming, etc. With the development of wireless technologies, WiFi, ZigBee, Bluetooth are widely utilized as potential solutions for providing one's location in GNSS-denied regions. However, multipath effects and electromagnetic interferences seriously cause signal attenuation, diffraction and refraction, 
thus degrading the quality of location information. Alternatively, an inertial measurement unit (IMU) consisting of triaxial gyroscopes and accelerometers senses the egomotion of the moving object and is free of external radio-frequency influences. Since MEMSIMU is self-contained, environment-independent and low-cost, it has been equipped in almost all the existing smart wearable devices for gait analysis, monitoring, sport statistics etc. [2]. However, the inertial sensor inevitably suffers from gyroscope drift and accelerometer bias, leading to the divergence of navigation solutions in a short period [3]. Great deals of research efforts have been made to enhance the navigation results. The most representative one is the zero velocity update (ZUPT) strategy [4, 5]. However, to some extent, it can only minimize the divergence speed of solutions. Using auxiliary sensors is another efficient way to compensate for the inertial sensor drift and bias. Unfortunately, for a PNS, the volume, weight and power consumption should be cautiously considered, thus the lighter and more compact hardware system is preferred. From the aspect of dead reckoning, the inertial instruments potentially imply the characteristics of pedestrian gait cycle, thus they can be naturally used as a step indicator for dead reckoning navigation with a pre-determined step length [6].

For the distance measuring with inertial sensors, two critical issues should be considered, i.e. step detection and step length estimation. For the step detection, a popular way is to use empirical acceleration thresholds in time domain [7]. Likewise, a given threshold can be applied to the differential specific force series [8]. [9] computes the variance and its duration information to judge one's step. The g-crossing algorithm counting from the rising-point of gravity is efficient and commonly employed as well [10, 11]. Alternatively, many efforts have been put in the frequency domain. The most representative one is proposed by Levi and Judd [12]. It substantially implements the fast Fourier transformation (FFT) to extract the frequency subsequently used for peak detection. Unfortunately, pseudo-peak seriously degrades its success rate. Under such framework, similar algorithms e.g. zero-crossing and autocorrelation are also developed in [6] and [13]. For the step length estimation, in the past few decades, the most common model is a bi-parametric linear model $[12,6]$. It is simple and only related to the step frequency. Recent years, apart from the step frequency, other parameters are introduced into the refined step length model as well, for instance, the acceleration variance [14], acceleration boundary [15, 16, 17], height
[18] and leg length [19]. These model parameters can be pre-calibrated analytically or trained with machine learning [20]. In addition, from the aspect of inertial navigation, a real-time step length estimation strategy is put forward to directly compute each step length with the aid of ZUPT [21]. However, it is more timeconsuming and has a higher computational complexity compared with other empirical step length models.

Above all, the aforementioned methods have two main drawbacks: (i) the thresholds and parameters in step detection are person-dependent and hence need to be tuned for different users and different motions. Otherwise, the success rate will be heavily degraded. (ii) Even if the parameters in these step length models are pre-determined, unique step length model still cannot adequately describe different motion behaviors, e.g. running and walking. Therefore, in this contribution, to overcome these drawbacks, an adaptive time- and frequency-domains joint distance measuring method is proposed by utilizing the means of behaviors classification. It consists of two critical issues: step detection and step length determination. Based on behavior classification, we propose a fourstage step detection strategy, where thresholds and step frequency are adaptively adjusted for different persons. For step length estimation, a two-segment functional model is established to accommodate different motion behaviors. This paper is arranged as follows: the distance measuring problem and the basic principle are described in Section 2. Pedestrian features and behaviors recognition are investigated in Section 3. Section 4 focuses on the step detection strategy based on behavior classification. A two-segment functional step length model is constructed in Section 5. Field tests are carried out to evaluate performances of our proposed method in Section 6. Finally, concluding remarks are drawn in Section 7.

\section{Problem formulation and background}

Step based pedestrian navigation system, in essence, is a typical dead reckoning system which recursively computes the one's position in real time by using two kinds of fundamental information during a motion cycle, i.e. moving distance and orientation. Usually, the orientation is acquired from the magnetometers, while the moving distance is indirectly measured by inertial sensors. The fundamental principle of pedestrian dead reckoning is generally interpreted in a defined plane by

$$
\left(\begin{array}{l}
x_{k} \\
y_{k}
\end{array}\right)=\left(\begin{array}{l}
x_{0} \\
y_{0}
\end{array}\right)+\sum_{i=1}^{k-1} d_{i}\left(\begin{array}{c}
\sin \theta_{i} \\
\cos \theta_{i}
\end{array}\right)
$$


where $\left(x_{k}, y_{k}\right)$ denotes the two-dimensional coordinate after the $k$-th motion cycle; $\left(x_{0}, y_{0}\right)$ denotes the initial location. $d_{i}$ and $\theta_{i}$ represent distance and moving orientation during the $i$-th motion period respectively. In the following, we will get an insight into how to accurately measure one's distance $d$ with triaxial accelerometers periodically. A wearable distance measuring device is particularly designed and developed with several modules including builtin BOSCH MEMS-IMU, STM32, Bluetooth, Lithium battery [22]. The chip model version 'nRF51822' integrates STM32 with Bluetooth module. Bluetooth low energy (BLE) 4.0 is applied to ensure the lowpower consumption for communications in real time. Also the collected IMU data is transmitted to cell-phone through the serial peripheral interface (SPI) for the implementation of the accumulated distance measuring procedure, which is mainly decomposed as two basic parts: step counting and step length determination. The maximum system sampling rate is $250 \mathrm{~Hz}$. The specifications of Lithium battery is $3.7 \mathrm{~V} 80 \mathrm{mAh}$. The device prototype is mounted on the surface of one's shoes. Figure 1 shows the top and slide views of the developed system.

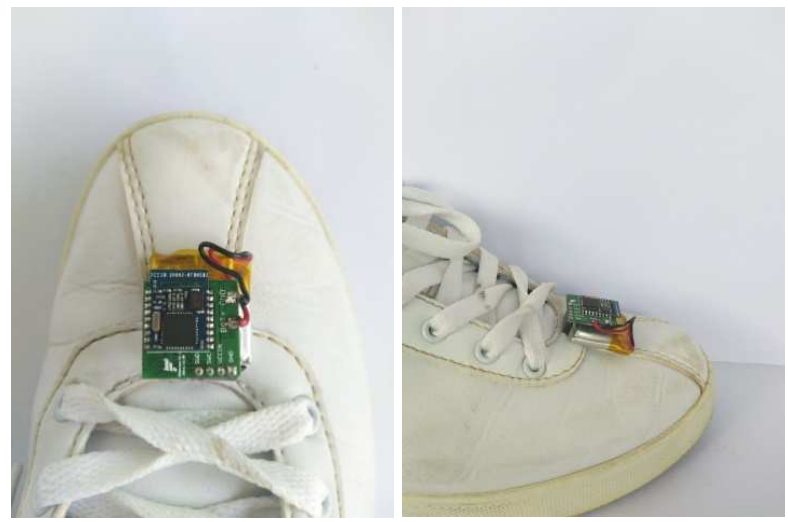

Fig. 1. The prototype of foot-mounted distance measuring system

\section{Pedestrian features and behaviors recognition}

In previous works, gaits information is extracted and recognized as steps mainly through fast Fourier transformation (FFT) and peak detection with thresholds and zero-crossing [23]. However, it is much more complicated in real applications accompanied with irregular motions (swings), strenuous exercise etc. These activities probably generate multiple pseudopeaks resulting in a risk of wrong step detection. Therefore the invariant frequency bandwidth would not be suitable for various activities. The activity classification performance is highly dependent on where the wearable device is mounted [24, 25, 26]. In this section, four sorts of activities including standing still, walking, running and irregular motions are considered and to be classified. Features in time and frequency domains can be utilized to identify the activities. For instance, the first and second moments, maximum, discrete cosine transformation coefficients, FFT coefficients, direct component, energy and entropy etc. $[27,28,29]$. Three of them i.e. variance, direct component (DC) and energy are jointly utilized to identify behaviors. For the $j$-th $j=1,2, \cdots, n$ sampled specific force in dataset $i$, its derived acceleration can be written such as

$$
a_{i, j}=\left\|l_{i, j}-b_{a}\right\|-\left\|g_{r}\right\|
$$

where $l$ represents the specific force vector measured by accelerometer; $g_{r}$ represents the reference gravity in the local frame, i.e. $\left[\begin{array}{lll}0 & 0 & g\end{array}\right]^{T} ; b_{a}$ denotes zero-bias term of accelerometer and can be approximated as

$$
b_{a} \approx \frac{1}{N} \sum_{s=1}^{N} l_{s}-g_{r}
$$

where $N$ is the number of static samples. Noting that the zero-bias term of IMU is estimated in a horizontal plane in a static mode, then it is parallel to the local frame of gr. Therefore we can reliably calculate the bias term $b_{a}$ by (3) in the body frame. It should be noted that after computing accelerations with (2), we still need to remove the influences of high-frequency noise from them. For this reason, the Hamming-window based, linear-phase finite impulse response (FIR) filter is introduced to generate the filtered acceleration $\tilde{a}$. Then it is rather easy to compute the variance $\sigma_{\tilde{a}_{i}}^{2}$. The DC component denoted as $f_{0}$ can be determined by using FFT and accordingly the energy of dataset $i$ is defined as [27]

$$
E_{\tilde{a}_{i}}=\frac{\sum_{j=1}^{n}\left|f_{j}\right|}{n}
$$

where $E$ represents the energy indicator; $f$ denotes the frequency after the DC component is removed. Figure 2 shows the three features values for different motion behaviors. It can be observed that these features are very effective for distinguishing the four behaviors. Based on the previous field tests, the thresholds are rather easy to be chosen empirically due to the features of the behaviors significantly differing from each other. Next we will investigate how to recognize the 

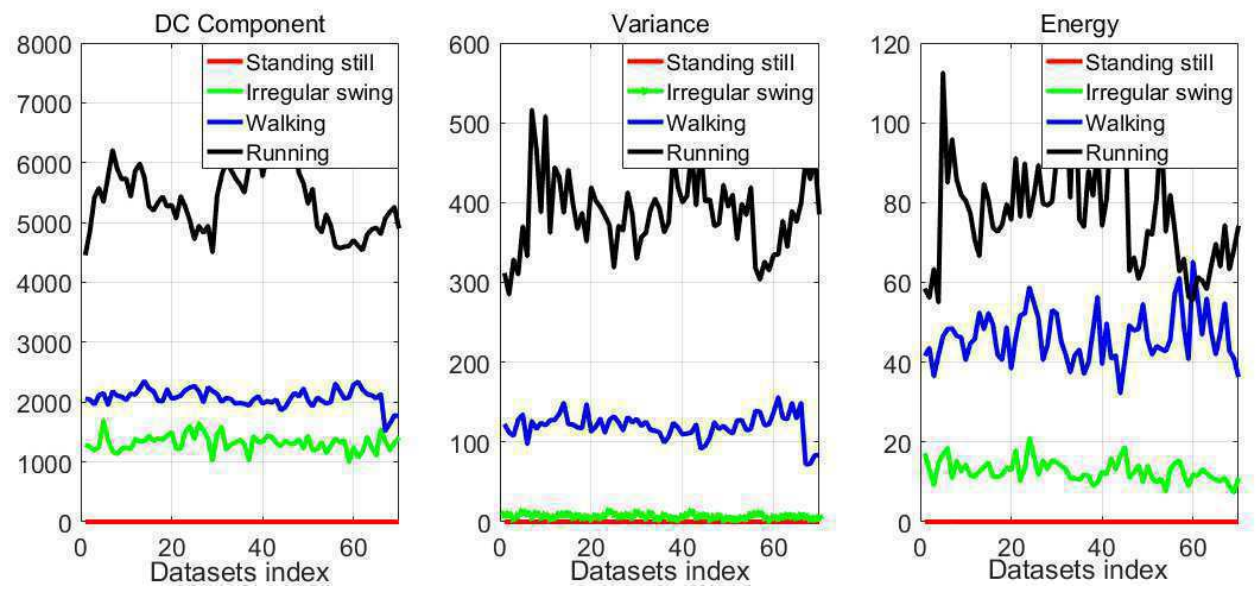

Fig. 2. Three features extracted from validation datasets

behavior based on the features information. A variety

of training algorithms have been applied to behavior

recognition, for instance, supported vector machine,

neural network, K-means and decision tree $[6,30]$.

In this paper, we propose the following real-time

state machine based multi-mode behaviors recognition

algorithm (see Figure 3). It is worthy to point out that

the behavior recognition is manipulated by making use

of the acceleration series within a two-second time

window in real time.
Table 1. Implementations of state machine based multi-mode behaviors recognition algorithm (one-cycle)

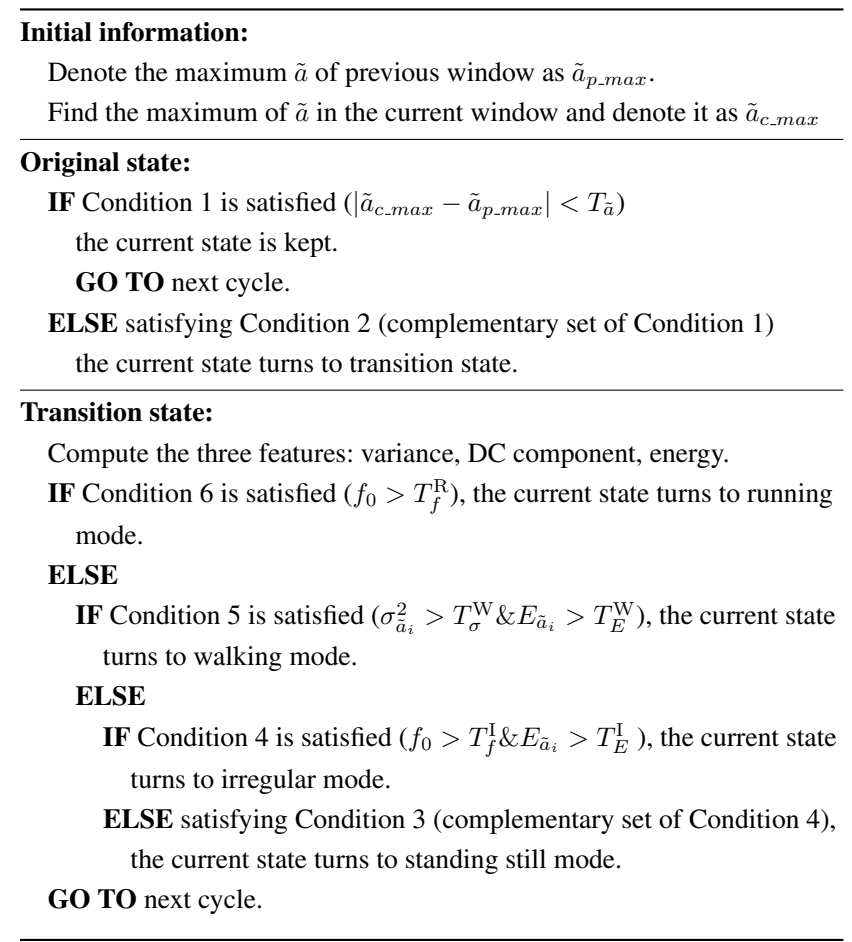

Table 1 depicts the implementation mechanism of our designed state machine, in detail. Note that symbol ' $T$ ' represents the threshold and its superscripts ' $\mathrm{R}$ ', ' $\mathrm{W}$ ' and ' $\mathrm{I}$ ' identify the corresponding behavior modes running, walking and irregular swing respectively. 


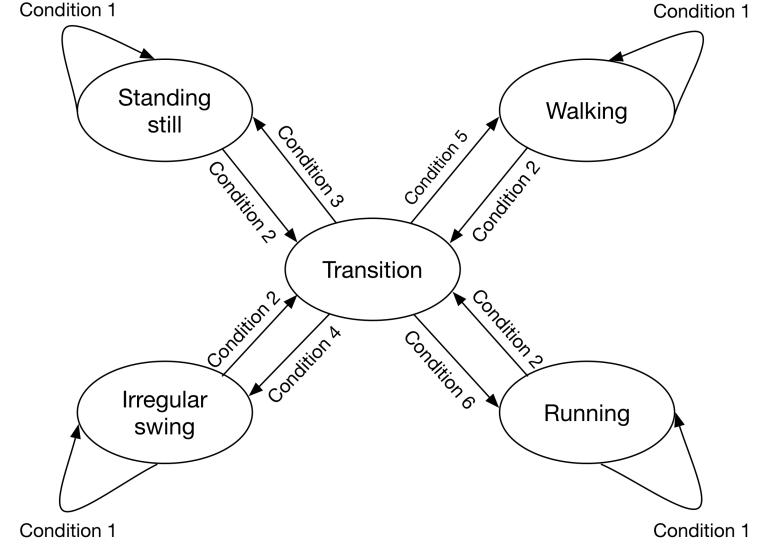

Fig. 3. Schematics of the designed state machine for behaviors recognition
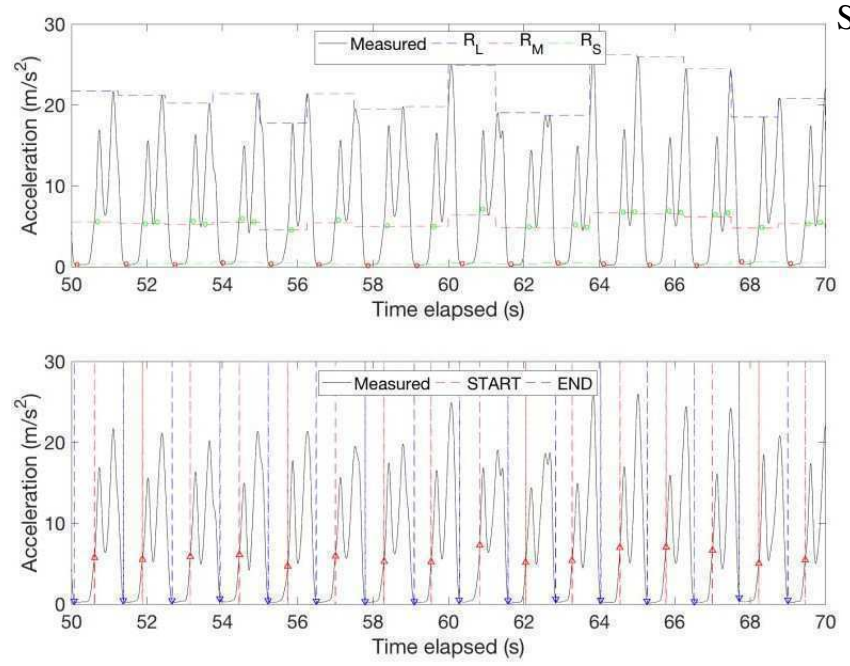

Fig. 4. The measured acceleration series with the generated reference lines $R_{\mathrm{L}}, R_{\mathrm{M}}$ and $R_{\mathrm{S}}$ (top); The measured acceleration series along with the step counting evidences (bottom).

\section{Time- and frequency- domain joint step detection}

Based on the behaviors identified in Section 3, a time- and frequency-domains joint step detection method is proposed. It needs to be clarified that for the step detection, once behavior is standing still or irregular swing, it will not be in the step counting process. In other words, only walking and running modes will trigger the step counting procedure. If one is in the transition state, we will count the step till the walking or running behavior is detected. The time resolution of behavior recognition is set as two seconds. The step counting procedure mainly involves four stages: FIR for walking and running data, adaptive window length determination, extrema points searching and cross-points identification. It needs to be pointed out that all the parameters in step detection are assigned with different configurations settings to suit for walking and running behaviors.

Stage 1: FIR for walking and running data

The parameters setting for FIR should be particularly configured for walking and running. Denoting $f_{\mathrm{A}}$ as sampling frequency, based on our previous sufficient tests (over 200 persons), the passband and stopband are set as $0 \sim 4 \mathrm{~Hz}$ and $4.5 \sim f_{\mathrm{A}} / 2 \mathrm{~Hz}$ respectively, while for running, these values are $0 \sim 10 \mathrm{~Hz}$ and $10.5 \sim f_{\mathrm{A}} / 2 \mathrm{~Hz}$ respectively.

Stage 2: Adaptive window length determination

Define $f_{\mathrm{D}}$ as the dominant frequency which is determined with following process: first we process acceleration data of a short-period through FFT then remove its DC component. Furthermore, an empirical threshold is introduced to avoid the influences from the high-order harmonics. Finally, the dominant frequency $f_{\mathrm{D}}$ is reliably acquired. To minimize the computation burdens for a stepping cycle, the window length is adaptively determined through

$$
w_{\tilde{a}}=\frac{f_{\mathrm{A}}}{f_{\mathrm{D}}}
$$

where $w_{\tilde{a}}$ represents the window length and varied with the dominant frequency.

\section{Stage 3: Extrema points searching}

Within the determined window by (5), we search the acceleration maxima and minima. Then these points with extremum are the candidates for identifying a pedestrian motion cycle. However, in real applications, due to pseudo-peaks, we additionally use thresholds $\left(T_{\mathrm{L}}, T_{\mathrm{S}}\right)$ to construct the following criterion to screen out the maxima and minima points

$$
\left\{\begin{array}{l}
\operatorname{maxima}:[\tilde{a}(p-1)<\tilde{a}(p)>\tilde{a}(p+1)] \&\left[\tilde{a}>T_{\mathrm{L}}\right] \\
\operatorname{minima:}[\tilde{a}(p-1)>\tilde{a}(p)<\tilde{a}(p+1)] \&\left[\tilde{a}>T_{\mathrm{S}}\right]
\end{array}\right.
$$

where $p=1,2, \ldots, w_{a_{i}}$.

\section{Stage 4: Cross-points identification}

Based on the extrema points confirmed above, we further introduce the 'cross-points' with the aid of three reference lines with values of $R_{\mathrm{L}}, R_{\mathrm{M}}$ and $R_{\mathrm{S}}$ (shown in Figure 4), since these cross-points 
are crucial to exhibit details of moving pace. Note that the symbol $\mathrm{R}$ represents the reference line; the subscripts ' $L$ ', ' $M$ ', ' $S$ ' correspond to 'large', 'median', 'small' respectively. They can be adaptively adjusted according to the maximum and minimum accelerations in a sliding window, which is flexible. The window size is selfdetermined based on the results of FFT analysis and there is no need to define the size manually. Given the current window index $i$, the reference lines are produced according to the following rules (see Table 2).

Table 2. Rules of producing $R_{\mathrm{L}}, R_{\mathrm{M}}$ and $R_{\mathrm{S}}$

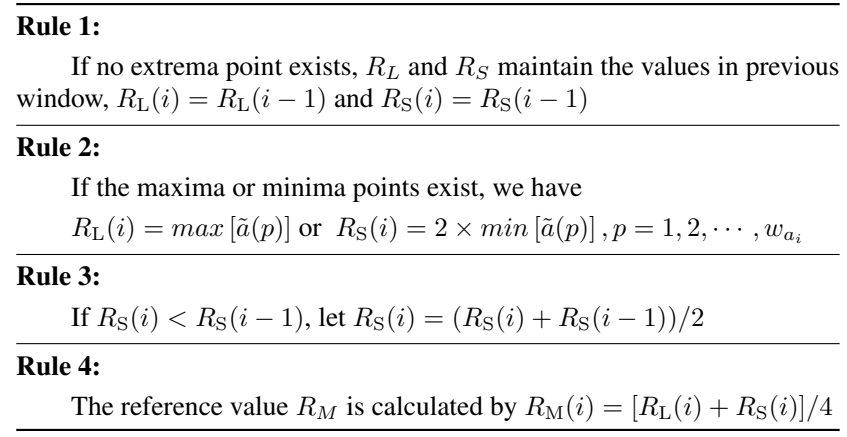

Meanwhile, with the established rules, we invent a simple step confirmation procedure as below. For convenience, 'START' and 'END' flags are marked for indicating a whole step interval.

(i) Search cross-points till the first rising cross-point on RM is found and mark it as 'START'.

(ii) Keep searching till the next descent cross-point on RS is found and mark it as 'END'.

(iii) Confirm the whole step cycle ranging from 'START' to 'END'.

(iv) Continue to seek a new rising cross-point on RM after the 'END' in (iii). Then mark it as a new 'START' and go to (ii).

\section{Two-segment functional based step length model}

A variety of step length models have been developed in the past few decades. These methods can be mainly categorized into the following three types: (i) Constant models. They usually contain a group of empirical personal parameters (e.g. height, gender) which require to be calibrated beforehand from person to person. It is time-invariant once the parameters are fixed, thus is rather simple and convenient to be applied. However, its accuracy cannot satisfy the high precision applications. (ii) Observation based models. Besides some empirical parameters mentioned in (i), the models also consist of inertial measurements, e.g. mean or minimum and maximum of accelerations. (iii) Indirect motion characteristics based models. Such models are usually constructed by some indirect motion information, typically e.g., step frequency. In general, models in (ii) and (iii) achieve comparative performances. To sum up, the representative models are given by Eqs. $(7) \sim(12)$,

$$
\begin{gathered}
d_{i}=K_{0}+K_{1} \times f_{S_{i}} \\
d_{i}=K_{0}+K_{1} \times f_{S_{i}}+K_{2} \times \sigma_{\tilde{a}_{i}}^{2} \\
d_{i}=K_{0}+K_{1} \times \frac{1}{f_{S_{i}}}+K_{2} \times \max \left(\tilde{a}_{i}\right) \\
d_{i}=K_{0} \times \sqrt[4]{\max \left(\tilde{a}_{i}\right)-\min \left(\tilde{a}_{i}\right)} \\
d_{i}=K_{0} \times h \times \sqrt{f_{S_{i}}} \\
d_{i}=K_{0} \times \sqrt[3]{\overline{\tilde{a}}_{i}}
\end{gathered}
$$

where $d_{i}$ indicates the step length of the $i$-th step period; $K_{0}, K_{1}, K_{2}$ are coefficients to be calibrated beforehand; $h$ denotes one's height; $\overline{\tilde{a}}_{i}$ denotes the mean value of $\tilde{a}_{i}$ during the $i$-th step period; $f_{S_{i}}$ indicates the step frequency during the $i$-th step period and is defined by

$$
f_{S_{i}}=\frac{1}{\Delta t_{i}}
$$

in which $\Delta t_{i}$ represents the duration of the $i$-th detected step. However, any individual model among them is not able to adequately describe different motion behaviors, e.g. walking and running. As a matter of fact, based on our tests, it is found that the models along with their parameters both need to be tuned according to different motions. Otherwise, the step length estimation will be inaccurate or even wrong. For instance, the step frequency contained models are more accurate in running scenarios, whereas they do not perform well in walking cases. On the contrary, the acceleration information shows its advantages on expressing the step length in running mode. For this reason, considering the merits and performances of all models above, the following two-segment functional model is constructed to estimate step length of running and walking separately:

$$
\left\{\begin{array}{cc}
d_{i}^{\mathrm{W}}=K_{0}^{\mathrm{W}}+K_{1}^{\mathrm{W}} \times \frac{1}{f_{S_{i}}}+ & K_{2}^{\mathrm{W}} \times \frac{1}{\sqrt{f_{S_{i}}}}+ \\
K_{3}^{\mathrm{W}} \times \max \left(\tilde{a}_{i}\right) & (\text { Walking) } \\
d_{i}^{\mathrm{R}}=K_{0}^{\mathrm{R}} \times \sqrt[3]{\overline{\tilde{a}}_{i}^{\mathrm{R}}} & \text { (Running) }
\end{array}\right.
$$




\section{Experiments and analysis}

In this section, pedestrian experiments are carried out to test the proposed method and fully evaluate its performance. The distance measuring system prototype is mounted on the left or right foot. The sampling rate is set as $200 \mathrm{~Hz}$ and the sensed data is transmitted to a smart phone through Bluetooth communication module in real time for latter distance estimation. Four pedestrian behaviors, including standing still, walking, running and irregular swing are all intentionally experienced during the pedestrian experiments. To examine the efficiency of behaviors recognition in Section 3, we present the related recognition results of one tested person (see Figure 5). It can be seen from this figure that the pedestrian samples contain these four sorts of behaviors and the 'true' behaviors are also marked there (top). The Green line indicates the 'on' and 'off' of transition state. When the behaviors change, the transition state 'on' is triggered and kept till a different activity occurs. In rare cases, transition state may be incorrectly triggered but wrong behaviour will not be determined, meanwhile the transition state quickly switches to the right behaviour mode. Thus, the identified behaviors by our method (see the bottom of Figure 5) are completely consistent with the true ones. Next we continue to validate the success rate of our step detection strategy based on the behaviors recognition results. 205 persons ( 78 are female and 127 are male) have been tested in the past few months. We classify the statistic results according to four different height ranges: (i) $<160 \mathrm{~cm}$ (42 persons); (ii) 161 $\sim 170 \mathrm{~cm}$ (85 persons); (iii) $171 \sim 180 \mathrm{~cm}$ (58 persons); (iv) $>180 \mathrm{~cm}$ (20 persons). Considering the space limitation, here we only list the detailed personal results of four representative persons in Table 3 where the 'WD' and 'RD' denote the reference walking and running distance respectively. The reference distance is acquired according to the standard track length of UESTC stadium which has the length of 400 meters for the innermost lane for one lap and 200 meters for half lap. All persons are required to move along with the trajectory of innermost lane. During the tests, the walking speed ranges $1 \sim 2 \mathrm{~m} / \mathrm{s}$ and the running speed ranges $2 \sim 4 \mathrm{~m} / \mathrm{s}$. The true total steps are manually counted by tested persons themselves.

Since the reference [12] has been extensively used in applications of step detection [11,10], we count the steps by using Levi and Judd's method in comparison with our proposed behaviour classification based timeand frequency-domains joint (BCTFD) step detection method as well. The statistical results for Levi and Judd's method and BCTFD method are respectively summarized in Tables 4 and 5. It is noted that overcounted and missed steps are both counted as wrong steps for success rate statistics.
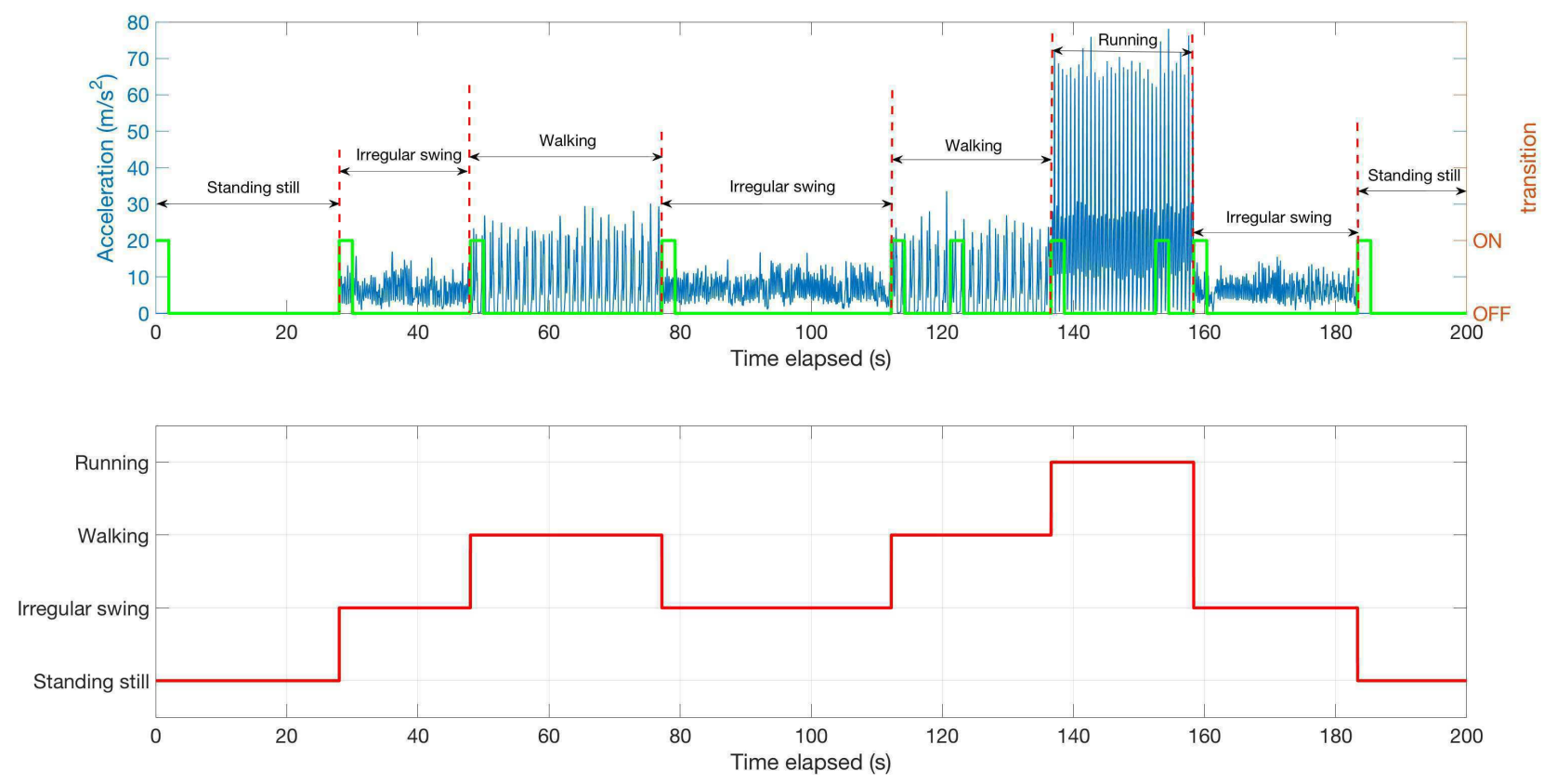

Fig. 5. The accelerations and identified behaviors 
Table 3. Pedestrian information of tested four persons

\begin{tabular}{cccccc}
\hline Person ID & Gender & $\begin{array}{c}\text { Height } \\
(\mathrm{cm})\end{array}$ & $\begin{array}{c}\text { Weight } \\
(\mathrm{kg})\end{array}$ & $\begin{array}{c}\text { WD } \\
(\mathrm{m})\end{array}$ & $\begin{array}{c}\text { RD } \\
(\mathrm{m})\end{array}$ \\
\hline PA & Male & 170 & 55 & 1200 & 800 \\
PB & Male & 180 & 70 & 1200 & 800 \\
PC & Female & 159 & 42 & 1200 & 800 \\
PD & Female & 158 & 61 & 1200 & 800 \\
\hline
\end{tabular}

Table 6. The average accuracy results for step detection (205 persons)

\begin{tabular}{ccc}
\hline Behaviors & Levi and Judd's method & BCTFD method \\
\hline Walking & $98.5 \%$ & $99.1 \%$ \\
Running & $97.1 \%$ & $98.5 \%$ \\
\hline
\end{tabular}

It is observed from Table 4 that for Levi and Judd's method, in general, the success rate of walking is slightly higher than running. The reason is that step signals during running are more likely suffered from the residual pseudo-peaks which cannot be entirely eliminated by FIR filter with only one invariant frequency-band. In fact, the frequency-band should be different for running and walking modes. Table 5 verifies such a point of view. Compared with statistics in Table 4, our proposed BCTFD method outperforms
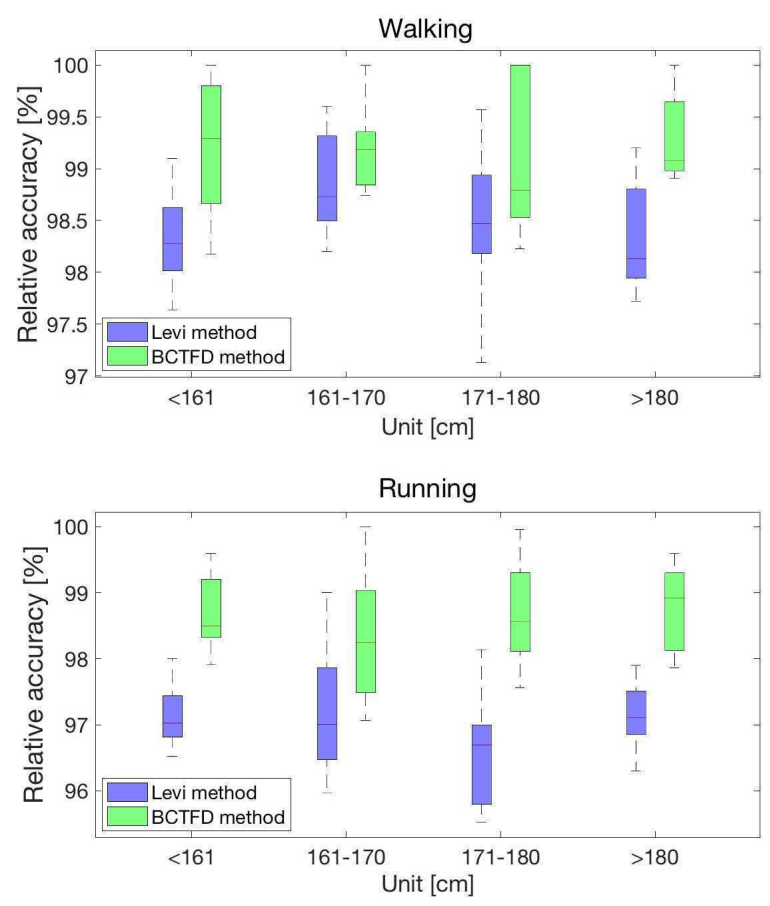

Fig. 6. The relative accuracy statistical results of step detection for tested 205 persons

Table 4. The step detection statistical results for Levi and Judd's method

\begin{tabular}{lccccccc}
\hline Behaviors & Person ID & True & Detected & Over-counted & Missed & Wrong & Success rate \\
\hline \multirow{6}{*}{ Walking } & PA & 891 & 887 & 6 & 10 & 16 & $98.2 \%$ \\
& PB & 747 & 741 & 0 & 6 & 6 & $99.2 \%$ \\
& PC & 877 & 881 & 10 & 6 & 16 & $98.2 \%$ \\
& PD & 870 & 870 & 4 & 4 & 8 & $99.1 \%$ \\
\hline \multirow{6}{*}{ Running } & PA & 384 & 380 & 0 & 4 & 4 & $99 \%$ \\
& PB & 349 & 338 & 0 & 11 & 11 & $96.8 \%$ \\
& PC & 470 & 460 & 0 & 10 & 10 & $97.9 \%$ \\
& PD & 401 & 393 & 0 & 8 & 8 & $98 \%$ \\
\hline
\end{tabular}

Table 5. The step detection statistical results for the proposed BCTFD method

\begin{tabular}{lccccccc}
\hline Behaviors & Person ID & True & Detected & Over-counted & Missed & Wrong & Success rate \\
\hline \multirow{6}{*}{ Walking } & PA & 891 & 891 & 0 & 0 & 0 & $100 \%$ \\
& PB & 747 & 747 & 0 & 0 & 0 & $100 \%$ \\
& PC & 877 & 878 & 1 & 0 & 1 & $99.8 \%$ \\
& PD & 870 & 871 & 1 & 0 & 1 & $99.8 \%$ \\
\hline \multirow{6}{*}{ Running } & PA & 384 & 384 & 0 & 0 & 0 & $100 \%$ \\
& PB & 349 & 350 & 1 & 0 & 1 & $99.7 \%$ \\
& PC & 470 & 474 & 4 & 0 & 4 & $99.1 \%$ \\
& PD & 401 & 404 & 3 & 0 & 3 & $99.2 \%$ \\
\hline
\end{tabular}


Table 7. The statistics of distance errors for M1 M7 (unit: m)

\begin{tabular}{|c|c|c|c|c|c|c|c|c|}
\hline Person ID & Behaviors & M1 & M2 & M3 & M4 & M5 & M6 & M7 \\
\hline \multirow{2}{*}{ PA } & Walking & 53.44 & 49.78 & 22.61 & 52.82 & 44.41 & 38.46 & 20.07 \\
\hline & Running & 55.87 & 35.54 & 34.17 & 37.79 & 57.56 & 31.11 & 20.46 \\
\hline \multirow{2}{*}{ PB } & Walking & 86.17 & 53.46 & 15.76 & 117.6 & 64.14 & 157.06 & 14.06 \\
\hline & Running & 72.52 & 70.14 & 70.73 & 43.29 & 64.83 & 49.75 & 19.7 \\
\hline \multirow{2}{*}{ PC } & Walking & 44.44 & 26.36 & 27.29 & 19.81 & 41.82 & 28.39 & 30.74 \\
\hline & Running & 53.72 & 51.15 & 51.15 & 50.91 & 53.22 & 50.05 & 46.95 \\
\hline \multirow{2}{*}{ PD } & Walking & 35.59 & 85.46 & 31.82 & 56.42 & 16.35 & 62.81 & 16.21 \\
\hline & Running & 124.63 & 128.34 & 80.53 & 65.86 & 122.89 & 55.73 & 50.44 \\
\hline
\end{tabular}

Table 8. The average distance relative errors for M1 M7 (205 persons)

\begin{tabular}{cccccccc}
\hline Behaviors & M1 & M2 & M3 & M4 & M5 & M6 & M7 \\
\hline Walking & $6.01 \%$ & $5.08 \%$ & $3.63 \%$ & $6.33 \%$ & $5.07 \%$ & $6.47 \%$ & $3.31 \%$ \\
Running & $12.1 \%$ & $9.9 \%$ & $11.1 \%$ & $8.32 \%$ & $12.56 \%$ & $7.83 \%$ & $6.32 \%$ \\
\hline
\end{tabular}
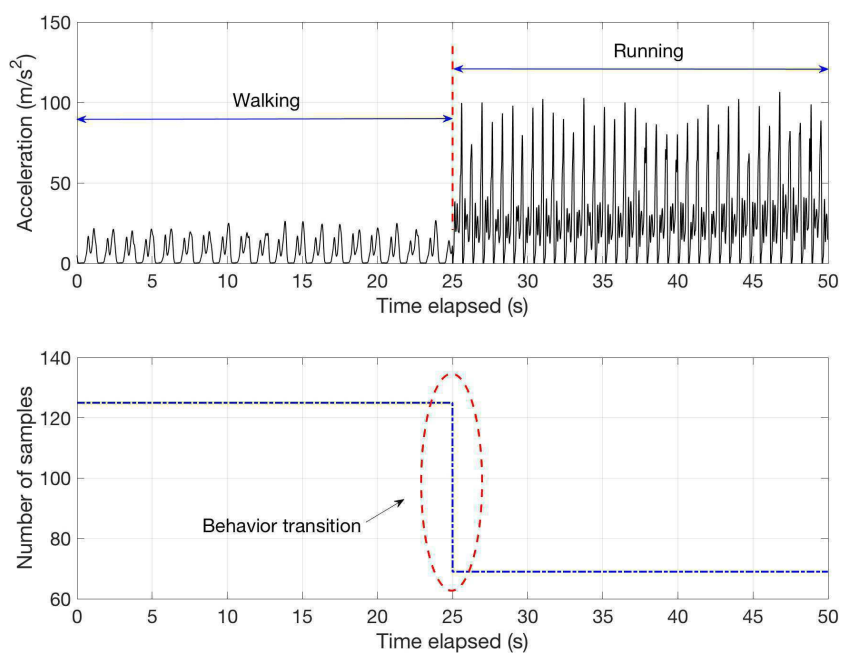

Fig. 7. The variation of window length corresponding with behavior transition

Levi and Judd's method. The relative accuracy of step detection for 205 persons is drawn with the boxplot for graphically depicting groups of tested persons through their quartiles (see Figure 6). Accordingly, the average accuracy results are given in Table 6. Overall, the performance of BCTFD method shows almost no difference between walking and running. Moreover, it exhibits better adaptability to all the tested persons in both walking and running behaviors. This is due to two aspects: behaviors classification and the step
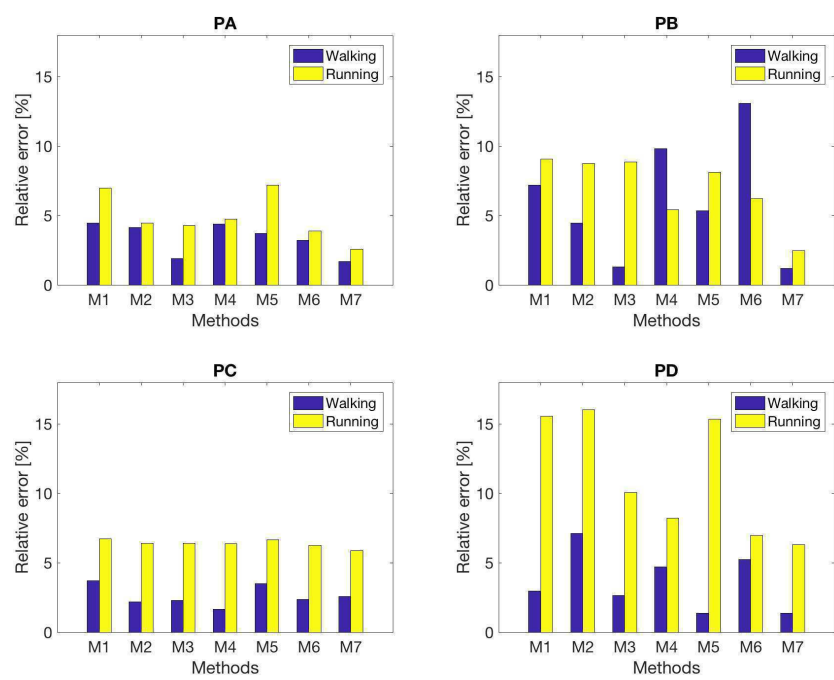

Fig. 8. The relative errors for all methods

identification strategy which in principle ensures the steps to be reliably confirmed. In addition, observed from Figure 6, no relationship can be found between step detection accuracy and one's height. Furthermore, we examine the window flexibility, which is strongly related to time and memory consumptions. Figure 7 clearly shows the variations of window length for different behaviors.

To evaluate the efficiency of step length models mentioned in Section 5, the walking and running distances (see Table 3) of all models are computed for 

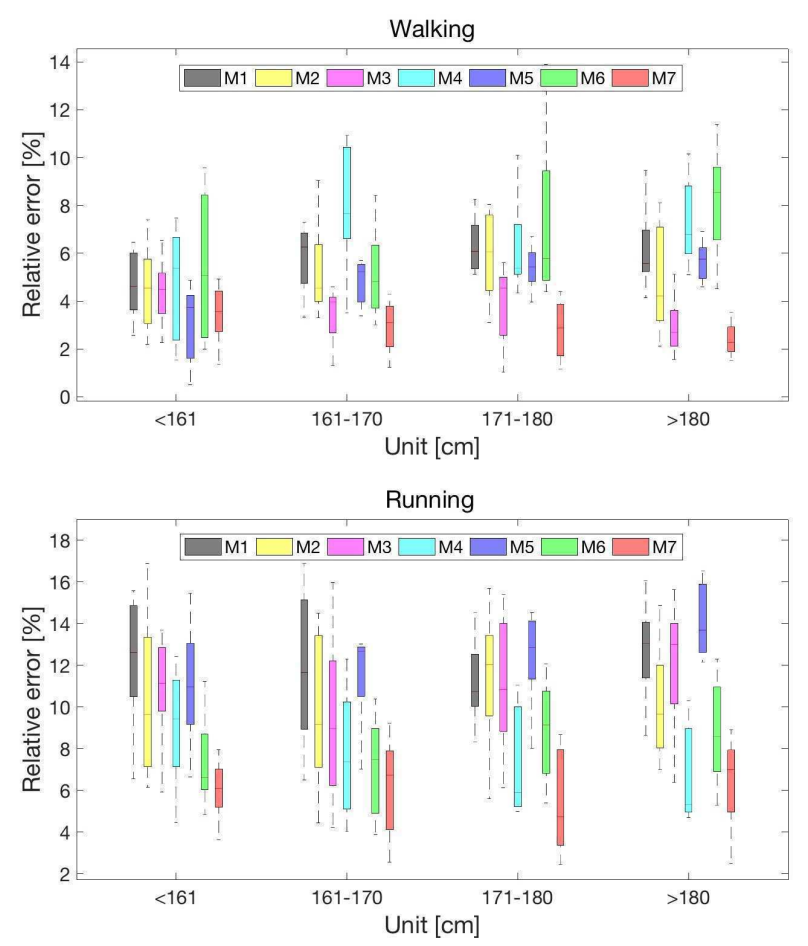

Fig. 9. The relative distance error statistical results for 205 tested persons

comparison purpose. Equations (7) (12) are denoted as M1 $\sim$ M6 and the proposed model in Eq. (14) is denoted as M7. Table 7 presents the error statistics of all methods and their relative errors are also drawn in Figure 8. The boxplot of the relative distance error for all tested persons are presented in Figure 9. The corresponding average distance relative errors statistics are also given in Table 8. It can be concluded that: in running case, M1, M2, M3 and M5 show extremely poor performances, especially for person PD. Similarly in walking case, M4 and M6 provide the unreliable distances particular for person PB. Compared with M1 $\sim$ M6, with the behaviors classification based twosegment function, distance estimated by M7 achieves the best accuracy, reliability and adaptability. More specifically, recalling the parameters in step length models for M1 $\sim$ M7, the following points can be made: (i) M5 with the height parameter shows no evident advantage compared with those methods without height. In other words, height parameter is not indispensable in the step length model. (ii) For walking behavior, the step frequency is the most crucial parameter for step length estimation. For example, the step frequency parameter is absent in M4 and M6, thus they produce the worst results. (iii) For running behavior, the acceleration is very critical for capturing ones motion. Without acceleration parameter, M1 and M5 generate the lowest step length accuracy.

\section{Conclusion}

In this paper, we develop a foot-mounted pedestrian distance measuring system. An adaptive time- and frequency-domains joint distance measuring method is proposed based on the behaviors classification. Behaviors recognition, step detection and step length estimation are all intensively investigated. Real experiments are carried out to demonstrate the vadility and efficiency of our proposed method compared with representative ones. The results show that our proposed method outperforms the existing representative methods and achieves the best accuracy, reliability and adaptability. Therefore, the proposed method is suitable and is of great benefit to pedestrian navigation applications. In the future, attention will be put on dead reckoning with the aid of auxiliary sensors [31, 32], e.g. magnetometers, barometers etc. On the other hand, more pedestrian behavior modes will be taken into account, such as going upstairs, downstairs, climbing and etc.

\section{Acknowledgement}

This work is supported by the National Natural Science Funds of China (Grant No. 41604025), the State Key Laboratory of Geodesy and Earth's Dynamics (Institute of Geodesy and Geophysics, CAS) with the Grant No. SKLGED2018-3-2-E, and the Fundamental Research Funds for the Central Universities (Grant No. ZYGX2018J080).

\section{REFERENCES}

1. Mezentsev O. and Lachapelle G., "Pedestrian dead reckoning a solution to navigation in GPS signal degraded areas," Geomatica, Vol. 59, No. 2, 5430-5434 (2005)

2. Godha S. and Lachapelle G., "Foot mounted inertial system for pedestrian navigation. Measurement Science and Technology," Vol. 19, No. 7, 075202(19) (2008)

3. Zhou Z., Li Y., Zhang J. and Rizos C., "Integrated navigation system for a low-cost quadrotor aerial vehicle in the presence of rotor influences," Journal of Surveying Engineering, Vol. 143, No. 1, 05016006(1-13) (2017) 
4. Nilsson J. O., Skog I. and Handel P. "Performance characterisation of foot-mounted ZUPTaided INSs and other related systems," 2010 International Conference on Indoor Positioning and Indoor navigation, IEEE, September, Zurich, Switzerland (2010)

5. Skog I., Hndel P., Nilsson J. O. and Rantakokko J., "Zero-velocity detection an algorithm evaluation," IEEE Transactions on Biomedical Engineering, Vol. 57, No. 11, 2657-2666 (2010)

6. Sun Z., Mao X., Tian W. and Zhang X. "Activity classification and dead reckoning for pedestrian navigation with wearable sensors," Measurement science and technology, Vol. 20, No. 1, 015203(110) (2009)

7. Cho S. Y. and Park C. G., "MEMS based pedestrian navigation system," The Journal of Navigation, Vol. 59, No. 1, 135-153 (2006)

8. Fang L., Antsaklis P. J., Montestruque L. A., Mcmickell M. B., Lemmon M. and Sun Y., et al. "Design of a wireless assisted pedestrian dead reckoning system - the NavMote experience," IEEE Transactions on Instrumentation \& Measurement, Vol. 54, No. 6, 2342-2358 (2005)

9. Yang L., Li Y. and Rizos C., "Walking status detection for pedestrian navigation" Proceedings of China Satellite Navigation Conference 2014: Lecture Notes in Electrical Engineering, Springer, Berlin, Heidelberg, Vol. III, 305, 485-497 (2014)

10. Leppkoski H., Collin J. and Takala J., "Pedestrian navigation based on inertial sensors, indoor map, and WLAN signals," Journal of Signal Processing Systems, Vol. 71, No. 3, 287-296 (2013)

11. Perttula A., Leppkoski H., Kirkko-Jaakkola M., Davidson P., Collin J. and Takala J., "Distributed indoor positioning system with inertial measurements and map matching," IEEE Transactions on Instrumentation and Measurement, Vol. 63, No. 11, 2682-2695 (2014)

12. Levi R. W. and Judd T., "Dead reckoning navigational system using accelerometer to measure foot impacts,” U.S. Patent 5583776. (1996)

13. Gusenbauer D, Isert C. and Krsche J., "Selfcontained indoor positioning on off-the-shelf mobile devices," 2010 International Conference on Indoor Positioning and Indoor navigation, IEEE, September, Zurich, Switzerland (2010)

14. Zhang H., Yuan W., Shen Q., Li T., and Chang $\mathrm{H}$., "A handheld inertial pedestrian navigation system with accurate step modes and device poses recognition,” IEEE Sensors Journal, Vol. 15, No. 3, 1421-1429 (2015)
15. Krach B. and Roberston P., "Cascaded estimation architecture for integration of foot-mounted inertial sensors," Position, Location and Navigation Symposium, 2008 IEEE/ION, IEEE, 112-119 (2008)

16. Li X., Wang J. and Liu C., "A Bluetooth/PDR integration algorithm for an indoor positioning system," Sensors, Vol. 15, No. 10, 24862-85 (2015)

17. Ngoc-Huynh H., Huu T. P. and Gu-Min J., "Stepdetection and adaptive step-length estimation for pedestrian dead-reckoning at various walking speeds using a smartphone," Sensors, Vol. 16, No.9, 1423(1-13) (2016)

18. Tian Q., Salcic Z., Wang I. K. and Pan Y., "A multi-mode dead reckoning system for pedestrian tracking using smartphones," IEEE Sensors Journal, Vol. 16, No.7, 2079-2093 (2016).

19. Lan K. C., Shih W. Y., "Using smart-phones and floor plans for indoor location tracking," IEEE Transactions on Human-Machine Systems, Vol. 44, No. 2, 211-221 (2014)

20. Sy C. and Cg P., "MEMS based pedestrian navigation system," Journal of Navigation, Vol. 59, 135-153 (2006)

21. Fourati H., "Heterogeneous data fusion algorithm for pedestrian navigation via foot-mounted inertial measurement unit and complementary filter," IEEE Transactions on Instrumentation and Measurement, Vol. 64, No. 1, 221-229 (2014)

22. Zhou Z., Mo S., Du S. and Geng J. "Behaviors recognition and step detection for pedestrian navigation via a foot-mounted inertial measurement unit," Proceedings of ION GNSS 2017, Portland, Oregon, 357-367 (2017)

23. Weimann F., Abwerzger G. and Hofmannwellenhof B., "A pedestrian navigation system for urban and indoor environments," Proceedings of ION GNSS 20th International Technical Meeting of the Satellite Division, September, Fort Worth, TX, 1380-1389 (2007)

24. Maurer U., Smailagic A., Siewiorek D. P. and Deisher M., "Activity recognition and monitoring using multiple sensors on different body positions," International Workshop on Wearable and Implantable Body Sensor Networks, IEEE Computer Society, 113-116 (2006).

25. Kawahara Y., Kurasawa H. and Morikawa H., "Recognizing user context using mobile handsets with acceleration sensors," IEEE International Conference on Portable Information Devices, IEEE, May, Orlando, FL, USA. (2007) 
26. Troped P. J., Oliveira M. S., Matthews C. E., Cromley E. K., Melly S. J. and Craig B. A., "Prediction of activity mode with global positioning system and accelerometer data," Medicine and Science in Sports and Exercise, Vol. 40, No. 5, 972-978 (2008)

27. Bao L. and Intille S., "Activity recognition from user-annotated acceleration data. International Conference on Pervasive Computing," Proceedings of Pervasive 2004, Lecture Notes in Computer Science, Vol. 3001, 1-17(2004)

28. He Z. and Jin L., "Activity recognition from acceleration data based on discrete consine transform and SVM," IEEE International Conference on Systems, Man and Cybernetics, IEEE, San Antonio, TX, USA 5041-5044 (2009)

29. Wang S., Chen Y. and Chen Z., "Recognizing transportation mode on mobile phone using probability fusion of extreme learning machines," International Journal of Uncertainty, Fuzziness and Knowledge-Based Systems, Vol. 21, No. 2, 13-22 (2013)

30. Deng W. Y., Zheng Q. H. and Wang Z. M., "Crossperson activity recognition using reduced kernel extreme learning machine," Neural Networks, Vol. 53, No. 5, 1-7 (2014)

31. Zhou Z., Li Y., Liu J. and Li G., "Equality constrained robust measurement fusion for adaptive Kalman-filter-based heterogeneous multi-sensor navigation," IEEE Transactions on Aerospace and Electronic Systems, Vol. 49, No. 4, 2146-2157 (2013)

32. Zhou Z., "Optimal batch distributed asynchronous multi-sensor fusion with feedback," IEEE Transactions on Aerospace and Electronic Systems, DOI: 10.1109/TAES.2018.2847979 (2018) 